\title{
Chediak-Higashi Syndrome Presented as Accelerated Phase in a 3 Year Girl Child: Case Report of a Rare Entity
}

Sen Rajeev ${ }^{1}$, Kataria Sant Prakash ${ }^{1}$, Agarwal Ruchi ${ }^{1 *}$, Chhabra Sonia ${ }^{1}$ and Mittal Kundan ${ }^{2}$

${ }^{1}$ Department of Pathology and Pediatrics, Pt. B.D Sharma Post Graduate University of Medical Sciences, Rohtak, India

${ }^{2}$ Department of Pathology, Bhagat Phool Singh, Government Medical College for Women, Khanpur Kalan, Sonepat, Haryana, India

\begin{abstract}
Chediak-Higashi Syndrome is a rare autosomal recessive disease, characterized by oculocutaneous albinism, recurrent pyogenic infections of skin, mucosa and respiratory system, prolonged bleeding time, easy bruisability and neurologic deficit. The hallmark of this syndrome is the presence of abnormal intracytoplasmic giant granules in al granule containing cells including leukocytes in blood and bone marrow.
\end{abstract}

We here report a case of Chediak-Higashi Syndrome in a 3 year old girl child who had recurrent chest infections, pancytopenia, hypopigmentation, lymphadenopathy and hepatosplenomegaly.

Keywords: Accelerated phase; Albinism; Chediak-Higashi syndrome; Primary granules; Lymphohistiocytic infiltration

\section{Introduction}

Chediak-Higashi Syndrome (CHS) is a rare autosomal recessive disorder of all lysosomal granule containing cells with clinical features involving the hematologic and neurologic systems. This immunodeficiency was first reported by Beguez Cesar in 1943 and then further described by Chediak in 1952 and Higashi in 1954 [1]. Morbidity results from patients succumbing to frequent bacterial infections or due to development of accelerated phase. The CHS gene was characterized in 1996 as the LYST (lysosomal trafficking regulator) or CHS 1 gene and is located on chromosome 1, q42-44. This gene effects protein synthesis or/and maintenance of storage and secretory lysosomal granules of leukocytes and fibroblasts, dense bodies of platelets, azurophilic granules of neutrophils and melanosomes of melanocytes [2].

\section{Case Report}

A 3 year old girl child presented with complaints of high grade fever associated with chills and rigor, dry cough for seven days, decreased intake and lethargy for two days. Past history of recurrent episodes of fever and chest infections were present since birth, approximately 5-6 episodes per year. There was no significant family history. Her developemental milestones were delayed.

On general physical examination, patient was febrile $\left(101.5^{\circ} \mathrm{F}\right)$, pulse- $110 / \mathrm{min}$ regular and respiratory rate- $22 / \mathrm{min}$. She was poorly built and anemic. There were multiple cervical and inguinal enlarged lymph nodes varying $1-1.5 \mathrm{~cm}$ in diameter which were firm, mobile and non-tender. There were multiple small patches of hypopigmentation on the skin of face, neck and upper part of chest wall (Figure 1). On systemic examination, chest was bilaterally clear and cardiovascular system was unremarkable. Liver was palpable $7 \mathrm{~cm}$ below costal margin with smooth surface and sharp margins. Splenomegaly was also present $6 \mathrm{~cm}$ below costal margin. Her gait was normal.

Laboratory investigations revealed hemoglobin-5 gm/dL, total leukocyte count- $4.9 \times 10^{3} / \mathrm{L}$, differential count-neutrophils $30 \%$, lymphocytes $64 \%$, monocytes $4 \%$, eosinophils $2 \%$, basophil $0 \%$, platelet count $-70 \times 10^{3} / \mathrm{L}$ and Erythrocyte Sedimentation Rate (ESR)-36 mm in 1st hour. Peripheral smear revealed normocytic normochromic to mild microcytic hypochromic picture; total leukocyte count was within normal range with lymphocytosis. Majority of the lymphocytes show large single granule in their cytoplasm (Figure 2). Bone marrow aspiration revealed hypercellularity with myeloid:erythroid ratio of 1:1. The cytoplasm of nucleated cells of trilineage precursors show dysplastic features along with abnormal giant purple coloured granulation. These granules were myeloperoxidase and sudan positive and negative for metachromasia by toludine blue. Phagocytic activity was increased. Iron store ranged from grade II to III. These findings suggested the diagnosis of Chediak-Higashi Syndrome.

Biochemical and urine examinations were within normal limits. Stool examination revealed presence of acinobacter species. Ultrasonography of the abdomen showed hepatomegaly and splenomegaly measuring $11.8 \mathrm{~cm}$ and $13.5 \mathrm{~cm}$ respectively with normal echotexture. Gall bladder, pancreas and both kidneys were normal. Few mesenteric lymph nodes and free fluid were also seen.

Patient was treated with paracetamol and amoxycillin for control of fever and infection. Packed cell transfusion and haematinics given for anemia. Hospital stay was uneventful and patient was discharged later on after 24 days without any complaints. She was advised for bone marrow transplantation but it could not be performed due to financial

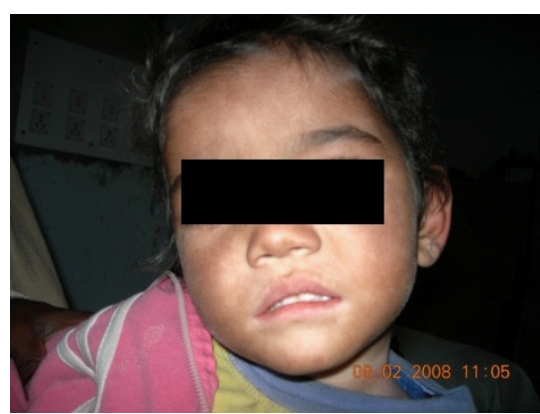

Figure 1: Photograph of the patient showing multiple hypopigmented patches on face.

*Corresponding author: Dr. Ruchi Agarwal, 673/35, Janta Colony, Rohtak 124001, Haryana, India, Tel: 09215340907; E-mail: pg.ruchi@gmail.com

Received December 28, 2012; Accepted April 27, 2013; Published April 29, 2013

Citation: Rajeev S, Prakash KS, Ruchi A, Sonia C, Kundan M (2013) ChediakHigashi Syndrome Presented as Accelerated Phase in a 3 Year Girl Child: Case Report of a Rare Entity. J Clin Case Rep 3: 275. doi:10.4172/2165-7920.1000275

Copyright: $\odot 2013$ Rajeev S, et al. This is an open-access article distributed under the terms of the Creative Commons Attribution License, which permits unrestricted use, distribution, and reproduction in any medium, provided the original author and source are credited. 


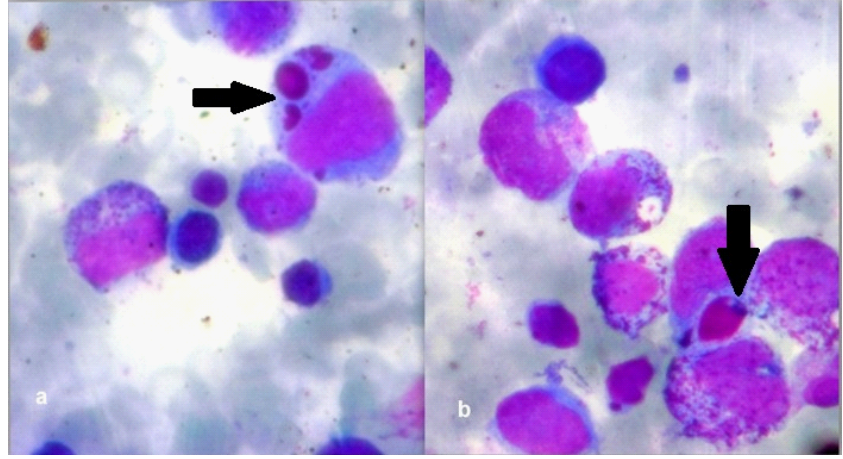

Figure 2: Photomicrograph of the bone marrow smears showing abnormal giant granules (arrows) in the precursor cells of leukocytes. (Romanowsky stain $X 400)$.

constraints. Patient regularly visited hospital for six months before she was lost during follow up.

\section{Discussion}

Chediak-Higashi Syndrome (CHS) is a rare inherited immunodeficiency disorder characterized by abnormal intracellular protein transport. This disorder can be considered a model for defects in vesicle formation, fusion or trafficking [1]. It is inherited as an autosomal recessive disorder with equal sex distribution [3].

The symptoms of CHS usually appear soon after birth or in children younger than 5 years. It is characterized by hypopigmentation of the skin, eyes and hair, recurrent pyogenic infections, abnormal natural killer cell function, prolonged bleeding time, easy bruisability and neurologic deficit.

Susceptibility to infections from phagocytic dysfunction ranges from mild recurrent skin infections to overwhelming fatal systemic infections. Affected patients are more susceptible to bacterial and fungal infections but have a normal resistance to viral infections. The infections mainly occur in the skin and respiratory tract, most frequently associated with Staphylococcus aureus and Streptococcus beta-haemolyticus [4]. Patients with CHS exhibit alterations in neutrophils including neutropenia, decreased deformability resulting in impaired chemotaxsis and delayed phagolysosomal fusion resulting in impaired bactericidal activity [1]. Natural killer cell function was also reduced [2]. In our case, onset of disease occurs in infancy and had history of recurrent infections.

Partial oculocutaneous albinism is prominent and associated with photophobia, rotary nystagmus and silvery colour of hairs. A neurologic manifestation in this syndrome includes abnormal gait, seizures, parasthesia, clumsiness, mental retardation and peripheral neuropathy [1]. Multiple areas of hypopigmentation were present in our case in face, neck and upper part of chest while neurologic manifestations were not detected, may be due to early detection of the disease. Thrombocytopenia is a common finding and may be the primary mechanism for bleeding tendency in the patient with CHS [5]. Death usually occurs in first decade of life due to recurrent infections, bleeding or development of accelerated lymphoma like phase, but survival up to second and third decades have been reported in few cases [6].

About $50-80 \%$ of patients of CHS enter into life threatening "accelerated phase" which is characterized by nonmalignant lymphohistiocytic infiltration of various organs such as liver, spleen, lymph nodes and bone marrow. It may occur shortly after birth or may be delayed for years, but invariably leads to death [3]. Accelerated phase is manifested by fever, jaundice, hepatosplenomegaly, lymphadenopathy, pancytopenia, bleeding disorders, abnormal liver function test, hypertriglyceredemia and overwhelming infections $[1,3]$. This lymphoma like stage is precipitated by viruses, particularly by Epstein-Barr virus infection [5]. Onset in early childhood mostly leads to development of an accelerated phase of the disease which is usually lethal without bone marrow transplantation. The adult form of CHS has a milder course, without any lymphohistiocytic infiltration [7]. In our case, the development of hepatosplenomegaly, lymphadenopathy, fever and pancytopenia suggest the onset of accelerated phase.

The hallmark of CHS is giant abnormal granules in all granule containing cells including melanocytes, peripheral leukocytes and their bone marrow precursors, central and peripheral nerve tissue, fibroblast and hair indicating that a common pathway in the synthesis of organelles responsible for storage is affected in these patients [1] These characteristic giant granules in all leukocytes result from abnormal fusion of both lysosomal azurophilic (primary) and specific (secondary) granules. Defective granules produce a dilute pigment which is responsible for partial albinism associated with CHS [3]. Similar findings were seen in the microscopic examination of peripheral smear and bone marrow aspirates of our patient.

Treatment options for CHS are limited and mainly symptomatic. Antibiotics are given to treat infections. High dose ascorbic acid $(200 \mathrm{mg} / \mathrm{kg})$ may improve the clinical course in some patients [2]. In accelerated phase, etoposside, steroids and intrathecal methotrexate have been tried [7]. The treatment of choice for CHS is Bone Marrow Transplantation (BMT) from a HLA compatible matched donor $[1,6]$. CHS patients not submitted to BMT; die at the mean age of 10 years [4].

Differential diagnosis of CHS includes other genetic forms of oculocutaneous albinism like Griscelli syndrome and Hermansky Pudlak syndrome, but both these syndromes lack the characteristic giant granules in neutrophils which are the hallmark of CHS. Acute and chronic myeloid leukemia may show giant granules resembling those seen in CHS, also referred to as pseudo-Chadiak Higashi anomaly [7].

The importance of careful examination of a well prepared and stained blood film by an experienced pathologist cannot be overemphasized. A high degree of suspicion is required to diagnose this syndrome on the basis of characteristic clinical features and the presence of giant granules in neutrophils. We emphasize on early diagnosis of this disease as early institution of only curative treatment in form of bone marrow transplantation can save patient's life.

\section{References}

1. Farhoudi A, Chavoshzadeh Z, Pourpak Z, Izadyar M, Gharagozlou M, et al. (2003) Report of six cases of chediak-higashi syndrome with regard to clinical and laboratory findings. Iran J Allergy Asthma Immunol 2: 189-192.

2. Siddiqui EU, Hanif S (2008) Chediak-Higashi Syndrome. Pak J Med Sci 24:328-30.

3. Islam AS, Hawsawi ZM, Islam MS, Ibrahim OA (2001) Chédiak-Higash syndrome: an accelerated phase with hereditary elliptocytosis: case report and review of the literature. Ann Saudi Med 21: 221-224.

4. Carnide EM, Jacob CM, Pastorino AC, Bellinati-Pires R, Costa MB, et al. (1998) Chédiak-Higashi syndrome: presentation of seven cases. Sao Paulo Med J 116: 1873-1878

5. Kapoor A, Munjal S, Arya R (2000) Chediak-Higashi syndrome--a case report Indian J Pathol Microbiol 43: 373-375. 
Citation: Rajeev S, Prakash KS, Ruchi A, Sonia C, Kundan M (2013) Chediak-Higashi Syndrome Presented as Accelerated Phase in a 3 Year Girl Child: Case Report of a Rare Entity. J Clin Case Rep 3: 275. doi:10.4172/2165-7920.1000275

6. Wolf J, Jacobi C, Breer H, Grau A (2006) Chediak-Higashi syndrome. Nervenarzt 77: 148, 150-152, 155-7.
7. Skubitz KM (2009) Qualitative disorders of leukocytes. In: Lee GR, Foerster

J, Lukens J, Paraskevas F, Geer JP (Eds.). Wintrobe's Clinical Hematology. (12thedn), Philadelphia, Williams and Wilkins 1548-1564. 